\title{
Visual search asymmetry for viewing direction
}

\author{
MICHAEL VON GRÜNAU and STÉPHANE DUBÉ \\ Concordia University, Montreal, Quebec, Canada
}

\begin{abstract}
In visual search experiments, we examined the existence of a search asymmetry for the direction with which three-dimensional objects are viewed. It was found that an upward-tilted target object among downward-tilted distracting objects was detected faster than when the orientation of target and distractors was reversed. This indicates that the early visual process regards objects tilted downward with respect to the observer as the situation that is more likely to be encountered. That is, the system is set up to expect to see the tops of these objects. We also found a visual field anisotropy, in that the asymmetry was more pronounced in the lower visual field. These findings are consistent with the idea that the tops of objects are usually situated in the lower visual field and less often in the upper field. Examination of the conditions under which the asymmetry and the anisotropy occur demonstrated the importance of the three-dimensional nature of the stimulus objects. Early visual processing thus makes use of heuristics that take into account specific relationships between the relative locations in space of the observer and 3-D objects.
\end{abstract}

One of the primary tasks that the visual system has to accomplish when it tries to make sense of a particular view of the world is to discriminate and identify objects. To accomplish this, the visual process first has to segregate visual surfaces in the three-dimensional (3-D) world. Since it is often not initially known where in the field of view the interesting or important objects are going to be, this initial process needs to take place across the whole visual field. It is also of importance that this first analysis occurs as rapidly as possible. These requirements put a lot of demands on the system. In order to facilitate the task, the primary process could be guided by later processes in a topdown fashion; these processes, including memory, might be better able to locate the kinds of objects and events that might be of importance (Wolfe, Cave, \& Franzel, 1989). In addition to this relatively short-term information, there are basic facts about the world in which we live that can restrict the possibilities or set clear probabilities, so that the task could be simplified. It seems that the visual system has evolved in exactly such a way as to have incorporated certain "basic truths" about the world. That is to say, certain assumptions can be made about the world that are usually true, and that do not have to be rediscovered over and over again (utilitarian theory of perception; Ramachandran, 1989).

Visual search tasks, in which observers search for a target among a variable number of distracting nontargets,

This work was partially supported by NSERC Operating Grant A53 to M.v.G. Partial reports of this work were presented at the annual meeting of the Canadian Society for Brain Behavior and Cognitive Sciences (1991-1992) and at the annual meeting of the Sociéte Québéquoise de Recherche en Psychologie (1991). Address correspondence to $\mathrm{M}$. von Grünau, Department of Psychology, Concordia University, 7141 Sherbrooke St. W., Montreal, PQ, Canada H4B 1R6.

Accepted by previous editor, Charles W. Eriksen can be used to demonstrate such heuristics in terms of search asymmetries (Treisman \& Gormican, 1988; Treisman \& Souther, 1985). That is, when target and nontarget stimuli are exchanged, the ease with which the target can be found may change dramatically. The many distracting stimuli provide the background against which the target either does or does not stand out. In other words, a target will be easily detected only when it displays an unusual situation seen against a background of a situation that is more often experienced. In this context, it has already been shown that light direction (illumination) is one such heuristic that the visual system can use (Enns \& Rensink, 1990; Ramachandran, 1988). Light from above, rather than from below, is the situation that is more likely to be encountered in our environment and is one that the search process seems to expect. For the perception of shape from shading, Kleffner and Ramachandran (1992) reported two assumptions that the visual system seems to have incorporated. The first one refers to a single light source interpretation, and the second to a light shining from above in relation to the retinal coordinates. Taken together, these observations suggest that there may be stages of processing very early in the visual system that are responsible for establishing feature links along a specific set of rules. Recently, Aks and Enns (1992) expressed the view that search is governed by a number of "quick and dirty" processes. This view argues that the preattentive visual system looks for predictors of object properties in the image, such as single light source and light direction (luminance gradient), rather than looking directly for objects.

In the present experiments, we examined viewing direction-whether an observer looks at objects from above or from below-with respect to a possible search asymmetry. We argue that it may constitute another heuristic that the early visual system can use to quickly analyze a given scene. Most objects that we deal with are 
smaller than ourselves and are usually located at heights below eye level, so we look down onto them. In other words, such 3-D objects would appear tilted downward from our point of view. From our results, it indeed appears that the more likely situation-the one expected by the early visual system-is one in which an observer looks at objects from above rather than from below. Furthermore, we show that this asymmetry is stronger in the lower visual field, where one would expect most of these objects to be located.

\section{EXPERIMENT 1}

In this experiment, detection of 3-D perspective objects tilted upward (so that they were viewed from below) or downward (viewed from above) was examined in a visual search task, in which they appeared among a background of oppositely tilted distractor objects. If our reasoning is correct, we expect to find a search asymmetry--upward-tilted objects would be detected faster than downward-tilted objects. This asymmetry should not be present with similar stimuli that do not appear as 3-D.

\section{Method}

Subjects. The observers consisted of the 2 authors, another experienced observer, and 7 naive volunteer subjects recruited among psychology students of Concordia University, all with normal or corrected-to-normal vision (20/20 Snellen).

Stimuli. For stimuli in the 3-D conditions, we used outline perspective blocks that were drawn in black $\left(1.78 \mathrm{~cd} / \mathrm{m}^{2}\right)$ on a white background $\left(71.9 \mathrm{~cd} / \mathrm{m}^{2}\right)$ and presented on the color monitor of a Macintosh II computer. As control stimuli, we used similarly drawn shapes without perspective (2-D conditions). Two examples of displays are presented in Figure 1. On the top, a downwardtilted target appears on a background of upward-tilted distractors; on the bottom, the situation is reversed.

The stimuli are also shown in Figure 3 in the top section of each graph. In this experiment, fixation was in the center of a rectangular area, $16^{\circ} \times 11^{\circ}$ of visual angle, within which the stimuli appeared. The stimuli each subtended $2^{\circ}$ of visual angle horizontally and vertically and were presented within an imaginary matrix of four columns and three rows with a random horizontal and vertical jitter of $\pm 0.5^{\circ}$ to avoid perfect alignment. The subjects were instructed to keep fixation throughout the experiment. This was monitored by the experimenter.

There were four conditions: two 3-D experimental and two 2-D controls (organization, or O). In condition "3D.down," the target block was tilted downward, while the distractor blocks were all tilted upward. In other words, the background was situated so that an observer would look upward at the stimuli, with the exception of the target stimulus. The situation was reversed in the "3D.up" condition. Here, the background was situated so that the observer would look down onto the stimuli, with the exception of the target, which would be viewed from below (stimulus direction, or D). In the 2-D conditions, the stimuli were redrawn, but all parallelograms were converted into rectangles to eliminate perspective cues and thus make them into flat shapes.

Procedure. The VSearch software (Enns, Ochs, \& Rensink, 1990) was used to control the experiment. Conditions were presented to each observer in a randomized order. Before the beginning of each condition, an observer was shown and familiarized with the target and distractor stimuli. We made sure that they readily perceived the 3-D stimuli as small 3-D blocks in the desired orientation. The observers also indicated that they did not perceive
$\mathbf{A}$

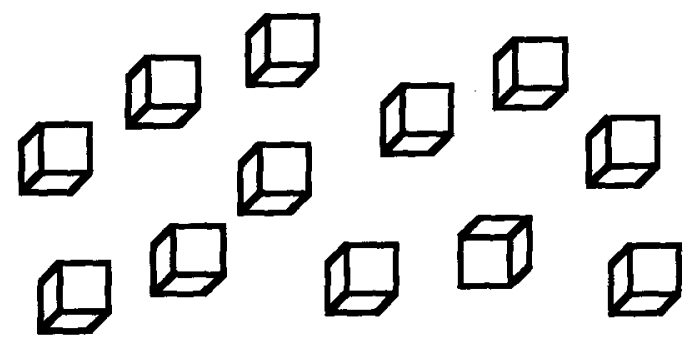

B

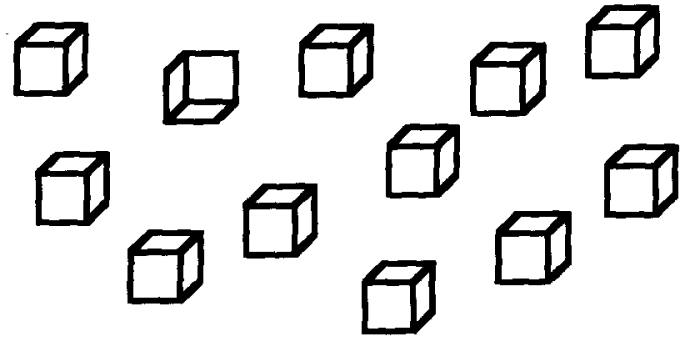

Figure 1. Examples of stimulus displays. A downward-tilted target cube among a background of upward-tilted distractor cubes (A), and an upward-tilted target cube among a background of downwardtilted distractor cubes (B).

the control stimuli as being 3-D. The observers received up to 60 practice trials (range: $30-60$ ). In each condition, experimental trials were presented in three blocks of 60 trials each. On half of the trials the target was present, and on the other half it was not. There were three display sizes $(1,6$, and 12 items). Altogether there were 30 repetitions for each of the six combinations. The order of presentation of display size and target presence was randomized by the program.

The observer's eye was positioned by a chin/foreheadrest at a distance of $57 \mathrm{~cm}$ from the screen, and he/she had to press keys to indicate, as quickly and accurately as possible, whether the target was present or absent. The stimuli remained on the screen until a response was given or up to a maximum of $2 \mathrm{sec}$. Both reaction times (RTs; to an accuracy of $1 \mathrm{msec}$ ) and errors (in percentages) were recorded. After each trial, the observer was shown either a small "+" or "-" in the center of the screen, indicating the correctness or incorrectness of the response. These also served as the fixation point for the next trial. After each block, which was initiated by the observer when ready, information was given as to the error rate. The subjects were instructed to try to keep errors below $10 \%$-that is, to slow down when the error rate was above $10 \%$ and to speed up when it was near $0 \%$.

\section{Results and Discussion}

For the analysis of the results, a four-factor withinsubjects analysis of variance (ANOVA) was performed on the RT data. The most interesting variables in the present context are the organization $(\mathrm{O})$ of the stimulus items--whether they were 2-D or 3-D, and the direction (D) of the stimulus items--whether the target was tilted downward or upward. Of specific interest is the $O \times D$ interaction, because it could indicate the existence of the expected asymmetry of target direction in the case of 
3-D, but not 2-D stimuli. This interaction was significant $[F(1,9)=5.39, p<.05]$ and is plotted in Figure 2. For the 2-D stimuli, mean RT did not differ as a function of stimulus direction $[F(1,9)=0.1, p>.76]$, but it did differ significantly for the 3-D stimuli $[F(1,9)=6.0, p<$ .04]. Mean RT was significantly faster when the target was tilted upward and presented among downwardtilted distractors. Thus, the expected search asymmetry for 3-D stimuli (but not 2-D stimuli) was found.

Since the factors of target present/absent $(P)$ and display size $(\mathrm{T})$ were also significant $[F(1,9)=39.51, p<$ .0005 for P; $F(2,18)=41.26, p<.0001$ for T], more detailed data are graphed in Figure 3. The RTs for targetpresent and target-absent trials were averaged for the 10 observers. These are displayed in Figure $3 \mathrm{~A}$ for the nonperspective 2-D controls and in Figure $3 \mathrm{~B}$ for the 3-D conditions as functions of the number of items in the display, with standard errors. The various stimuli are presented above the graphs for the different conditions. The results show a clear asymmetry for the direction of the stimuli in the 3-D conditions, but none for the controls, which was discussed above. In addition, it is also clear that target presence (filled symbols) resulted in faster RTs than target absence (open symbols) for all conditions. A larger display size resulted in slower RTs for all conditions, but the strength of this effect depended on stimulus direction in the 3-D conditions, as already discussed. These latter effects of target presence and display size are common in search tasks of this type and have been reported previously (e.g., Treisman \& Gormican, 1988).

To illustrate this last point in a different way, the slopes (processing times per item) are graphed in Figure $3 \mathrm{C}$ for all the conditions. Even though this measure might not be indicative for different underlying processing strategies (Green, 1991; Townsend, 1972), it can be used to summarize the present data. The slopes were

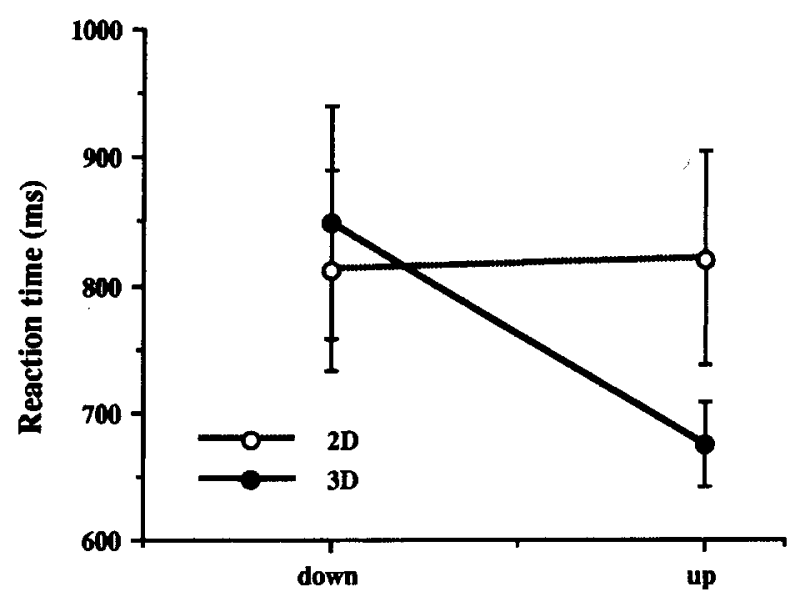

Stimulus direction

Figure 2. The asymmetry of stimulus direction (tilted downward vs. tilted upward) for 3-D cubes and 2-D comparison stimuli. Reaction time depends on stimulus direction only for 3-D stimuli. Means for 10 observers are graphed with their standard errors. calculated from the RTs by linear regression. The 2-D control conditions gave the steepest slopes [three-factor within-subjects ANOVA: $F(1,9)=6.46, p<.032]$, indicating that processing was most difficult in this case. There was no difference between the two 2-D conditions $[F(1,9)=0.01, p>.9]$. The ease of processing in the 3-D perspective conditions depended on which stimulus was the target and which was the distractor $[F(1,9)=7.03$, $p<.03]$. That is, this result again shows the existence of the search asymmetry for 3-D stimuli-processing was significantly easier when the target was tilted upward and the distractors downward, as compared with the reversed orientation.

The observed slope for the condition with the upwardtilted 3-D target was $16.0 \mathrm{msec}$ per item (target present), which might be considered a bit long for a preattentive process, where the cutoff point is usually (but arbitrarily) taken to be $10 \mathrm{msec}$ per item (e.g., Treisman \& Gormican, 1988). Others have reported shorter search times for similar objects (e.g., $7 \mathrm{msec}$; Enns \& Rensink, 1991), but their objects were also more elongated. In the same study, shorter but rotated cubes resulted in very similar slopes (16 msec per item). When comparing downward- and upward-tilted targets, processing time reduced from 32 to $16 \mathrm{msec}$ per item in the 3 -D condition of the present experiment. This is not incompatible with a change from attentive to preattentive processing, as it has been used in the past. We prefer instead to speak in terms of processing efficiency. The task of finding an upward-tilted object among a number of downwardtilted objects seems to be significantly easier than the reverse situation.

In addition to the RTs, Figures $3 \mathrm{~A}$ and $3 \mathrm{~B}$ also show the percent errors that were obtained in the different conditions. They were usually below $10 \%$, except in some cases for the larger display sizes, which is opposite to what would be expected if a speed-accuracy tradeoff had been present. Increased RTs, therefore, seem indicative of the requirement for genuinely longer processing times and not a change in search strategy.

The present results indicate that the speed of search for 3-D perspective objects depended on the orientation of these objects in 3-D space. The observed search asymmetry can also be interpreted in terms of an observer's viewing direction of the objects: When the target is viewed from below (i.e., is tilted upward) among distractors viewed from above, detection is faster than in the reverse situation. Thus, speed of detection was based on the relative position of objects with respect to the observer. It might be concluded that the present results are evidence for a (built-in or acquired) assumption of the early visual system that small objects are usually viewed from above. In a majority of cases, such an assumption would result in fast and correct detection of the object, and only rarely in an error. This heuristic, then, would improve the efficiency of the early visual system at the expense of the occasional mistake.

One clear prediction that one could derive from the above argumentation is that the obtained asymmetry 


\section{A: 2D non-perspective controls} down<smiles>C1C2CC3C1C1CCC3C21</smiles><smiles>C1C2C3C4CC5C3C1C5C24</smiles>

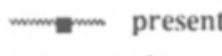<smiles>CO[13CH2]CC[13CH2]</smiles>
up<smiles>C1C2CC3C1C1CCC3C21</smiles>

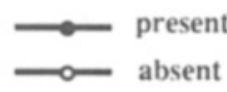

up

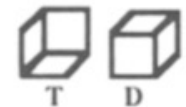

$\longrightarrow$ present

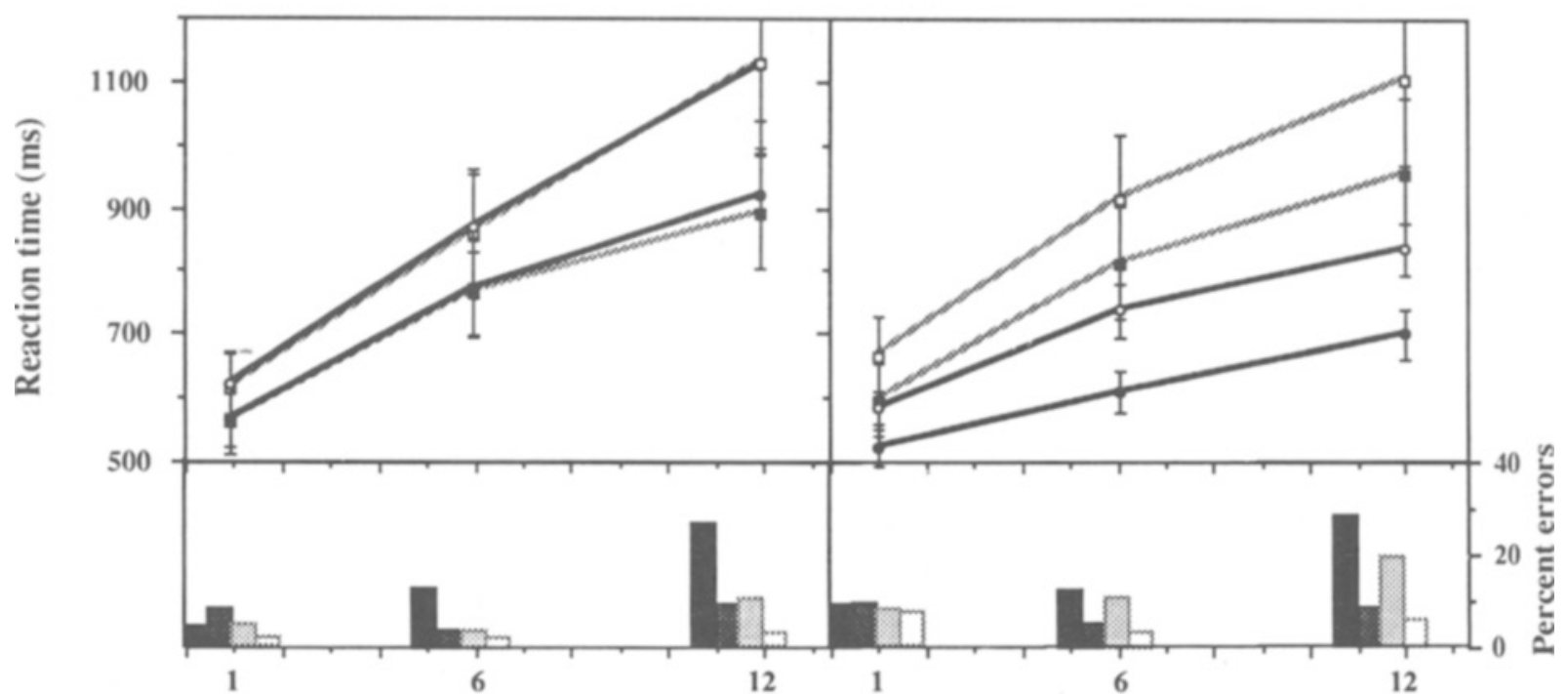

Display size

Display size

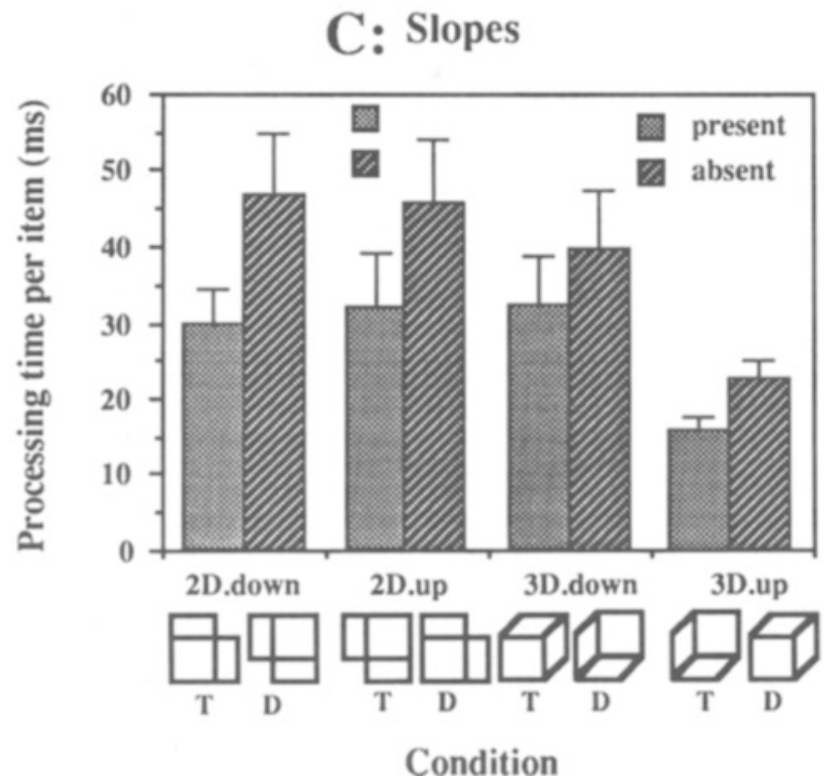

Figure 3. Reaction time as a function of display size for 2-D nonperspective controls (A) and 3-D perspective cubes (B). Results are graphed for the target-present and target-absent cases for conditions with downward- and upward-tilted targets. The stimuli are shown at the top of the graphs. Also included are the percentages of errors. The two darker columns indicate the "up" conditions, and the two lighter ones indicate the "down" conditions - the first one always for target present. Panel C shows slopes (processing times per item) for all the above functions, determined by linear regression. All error bars are $\pm 1 S E$. 
would be expected to be more pronounced in the lower visual field (LVF) than in the upper visual field (UVF). This is because a human observer would be expected to encounter small 3-D objects most often at heights below eye level. Their locations, therefore, would be predominantly in the LVF and only occasionally in the UVF. A well-adapted visual system, then, would profit most from the advantages of this asymmetry if it were confined to the LVF. In the UVF, the asymmetry might be reduced, might not be present, or could even be reversed.

To test for this anisotropy, we examined the present data in a different way. We partitioned the RT data with respect to the $y$ location of the target, either below or above the central fixation point, and performed a threefactor within-subjects ANOVA with organization $(\mathrm{O}$; 2-D vs. 3-D), direction (D; target down vs. up), and visual field (F; lower vs. upper). Since the asymmetry was the same for the target-present and target-absent conditions [ $\mathrm{O} \times \mathrm{D} \times \mathrm{P}$ interaction was not significant: $F(1,9)=$ $0.63, p>.44]$, the following analyses were performed only on the target-present data. (It would also not have been possible to categorize trials without target in terms of visual field location.) If the asymmetry of stimulus direction depended on the visual field, one would expect to find a significant $\mathrm{D} \times \mathrm{F}$ interaction. This was indeed the case $[F(1,9)=7.25, p<.025]$; it is plotted in Figure 4 . In both visual fields, an upward-tilted target could be detected faster than a downward-tilted target, but this effect reached significance only for the LVF $[F(1,9)=$ $7.43, p<.025]$ and not for the UVF $[F(1,9)=3.76, p>$ $.08]$. This is evidence for the existence of a visual field anisotropy with respect to the visual search asymmetry of stimulus direction.

As a control, we also partitioned the present data with respect to the $x$ location of the target and performed an analysis that was similar to the previous one. We thus

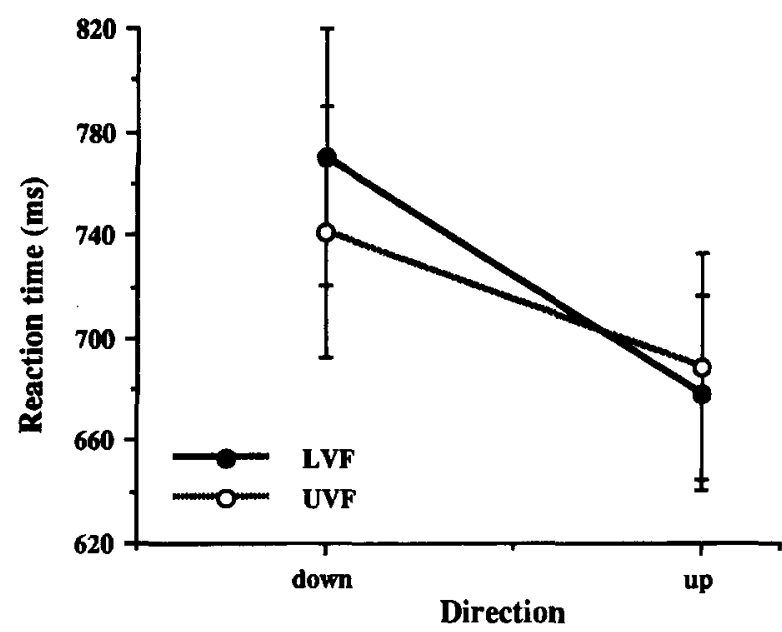

Figure 4. The asymmetry of viewing direction for targets located in the lower visual field (LVF) or the upper visual field (UVF). Only the LVF effect was significant. Means are graphed with their standard errors. obtained results for the left and right visual fields. No significant $\mathrm{D} \times \mathrm{F}$ interaction was found $[F(1,9)=1.4$, $p>.26]$, but there was an overall anisotropy-the responses for targets in the LVF were slightly faster than those for targets in the RVF $[F(1,9)=8.15, p<.019]$. The meaning of this anisotropy is not clear at the moment, but it could be related to a bias to examine a scene from left to right, following our experience with reading.

\section{EXPERIMENT 2}

The results of Experiment 1 may be interpreted as demonstrating that a specific viewing direction-looking at 3-D objects from above-is a normal situation for the early visual process. The argument of a built-in (or acquired) asymmetry was further strengthened by the fact that the asymmetry of viewing direction appeared more strongly in the LVF, where the objects would usually be located. The UVF, on the other hand, would deal with such objects much less often; accordingly, we did not find a significant asymmetry there, but a trend for the same asymmetry was nonetheless present. Since this question is of considerable interest, we repeated the first experiment using only the $3-D$ stimuli, presenting them either only in the LVF or only in the UVF.

\section{Method}

Subjects. The observers included the 2 authors and 13 undergraduate students in psychology, all with normal or corrected-tonormal vision (20/20 Snellen).

Stimuli and Procedure. The stimuli and procedure were the same as those in the first experiment. This time, however, the stimulus area was restricted to a rectangle of $16^{\circ} \times 5.5^{\circ}$ of visual angle in the center of the screen (the vertical extent was therefore reduced by half). Just above and below this area, centered horizontally, two fixation crosses were positioned on the screen. For the LVF conditions, the upper cross was fixated, and for the UVF conditions it was the lower cross. Fixation was monitored carefully by the experimenter during the experiment. Thus, in each condition, all the stimuli, the target, and the distractors were presented in one hemifield. Only 3-D perspective stimuli were used, with the target tilted either downward or upward. These four $(2 \times 2)$ conditions were presented to each observer in a different random order.

\section{Results and Discussion}

For the data analysis, RTs were averaged over all observers, and the slopes of the RT versus display size relationships were calculated as before. These results are displayed in Figure 5 for target-present and target-absent trials separately for the LVF and UVF conditions. Figure 5 also shows percent-error data for all conditions, including the factors of target present/absent and display size. Again, there was no evidence for a speed-accuracy tradeoff for any condition. Generally, errors occurred in less than $10 \%$ of the trials, and were higher only in some of the conditions with larger display sizes. Thus, in those cases, RT and error scores increased together.

A four-factor within-subject ANOVA on the RTs was performed. Unlike the corresponding results in Experi- 
A: Lower visual field

down
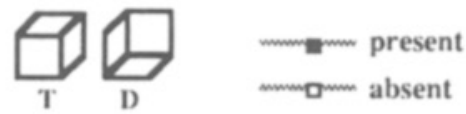

up
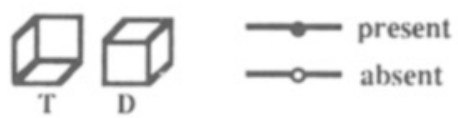

\section{B: Upper visual field}

down
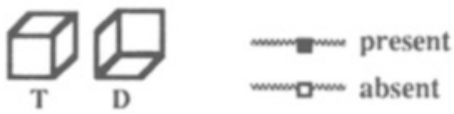

up
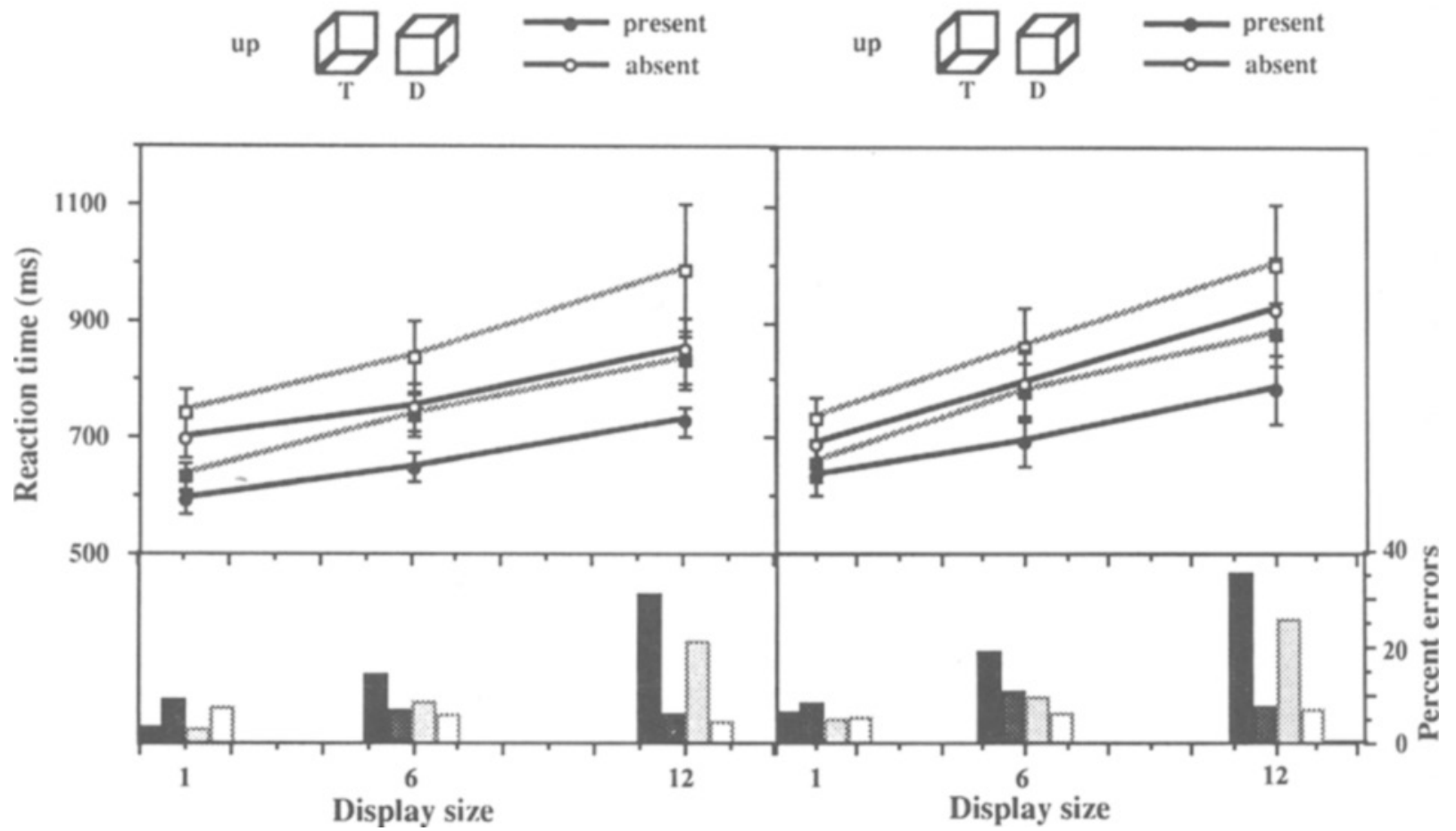

Figure 5. Reaction time as a function of display size for lower visual field (A) and upper visual field (B) presentations for targets tilted downward or upward. Results are graphed for the target-present and target-absent cases. Also included are the percentages of errors. The conventions are as for Figure 3. Error bars are $\pm 1 S E$.

ment 1 , there was no effect of visual field $[F(1,14)=$ $0.24, p>.63$ ], and no interaction with stimulus direction $[F(1,14)=0.17, p>.69]$. Stimulus direction, however, was significant $[F(1,14)=10.73, p<.006]$, indicating that the search asymmetry for stimulus direction that was found in Experiment 1 was present here in both the LVF and UVF in about the same strength. When the stimuli were presented only in one visual field, the task was significantly easier when the target was tilted upward and all the distractors were tilted downward. That is, in both the LVF and UVF, looking down at objects is the arrangement preferred by early vision. Figures $5 \mathrm{~A}$ and $5 \mathrm{~B}$ also display the significant effects of target present/absent $[F(1,14)=15.62, p<.0015]$ and display size $[F(2,28)=28.54, p<.0001]$. To facilitate comparisons to Experiment 1 (see General Discussion), it is worthwhile to mention that in terms of overall RT, responses to stimuli located in the LVF ( $750 \mathrm{msec})$ were somewhat faster than those to stimuli in the UVF $(770 \mathrm{msec})$, though not significantly so. Similarly, the asymmetry effect of stimulus direction was slightly (but not significantly) stronger in the LVF ( $88 \mathrm{msec}$ ) as compared with the UVF $(67 \mathrm{msec})$.

The present experiment replicated the search asymmetry of stimulus direction (or viewing direction) that was found in Experiment 1. A difference between the visual fields in terms of this asymmetry, on the other hand, could not be confirmed. To evaluate the possible anisotropy, it is necessary to consider that the distribution of targets and distractors differed in important aspects in the two experiments, which will be discussed below.

\section{EXPERIMENT 3}

The results of Experiments 1 and 2 indicated the existence of a specific effect of viewing direction - that is, looking at 3-D objects from above resulted in faster search rates. One reason for the absence of an interaction between the two visual fields and the search asymmetry in Experiment 2 could have been the narrow display that was used there. In a third experiment, the display was therefore extended to cover a larger area, which allowed 
for the stimulation of the same area as that in Experiment 1 in each hemifield.

\section{Method}

Subjects. The observers included the 2 authors and 5 undergraduate students in psychology, all with normal or corrected-tonormal vision (20/20 Snellen).

Stimuli and Procedure. The procedure that was used in the preceding experiments was followed, with the exception that the stimulated area was extended to cover a rectangle of $16^{\circ} \times 11^{\circ}$ of visual angle in the center of the screen. To keep their relative size constant, the individual stimuli were increased to twice the size of those in the previous displays $-4^{\circ}$ of visual angle horizontally and vertically (see Figure 6A). The display size was also modified to 2,6 , and 10 stimuli. Just above and below the display area, centered horizontally, two fixation crosses were displayed on the screen. For the LVF conditions the upper cross was fixated, and for the UVF conditions the lower cross was fixated. Fixation was again monitored carefully by the experimenter during the experiment. Only 3-D perspective stimuli were used, with the target tilted either downward or upward. These four $(2 \times 2)$ conditions were presented to the observers in different randomized orders.

\section{Results and Discussion}

For the data analysis, a four-factor within-subject ANOVA was performed on the RT. As usual, the effects of target present/absent $[F(1,6)=16.21, p<.007]$ and display size $[F(2,12)=14.97, p<.001]$ were significant. On the other hand, viewing direction $[F(1,6)=1.04, p>$ $.35]$, visual field $[F(1,6)=2.37, p>.17]$, and the corresponding interaction $[F(1,6)=1.57, p>.26]$ did not

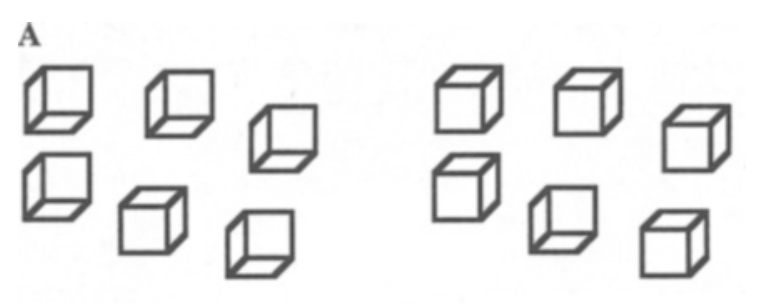

B

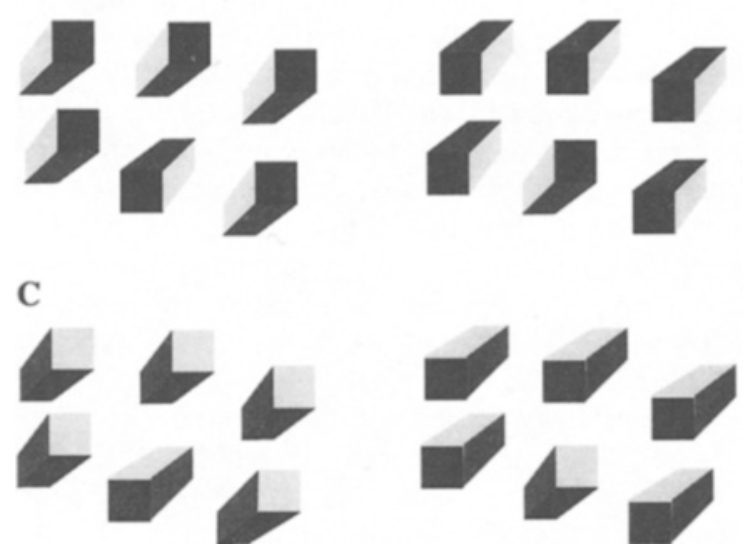

Figure 6. Stimuli used in Experiment 3 (A), Experiment 4, Part 1 (B), and Experiment 4, Part 2 (C). All stimuli were about $4^{\circ} \times 4^{\circ}$ of visual angle. On the left, a downward-tilted target is among upwardtilted distractors. On the right, an upward-tilted target is surrounded by downward-tilted distractors. Display size is 6.

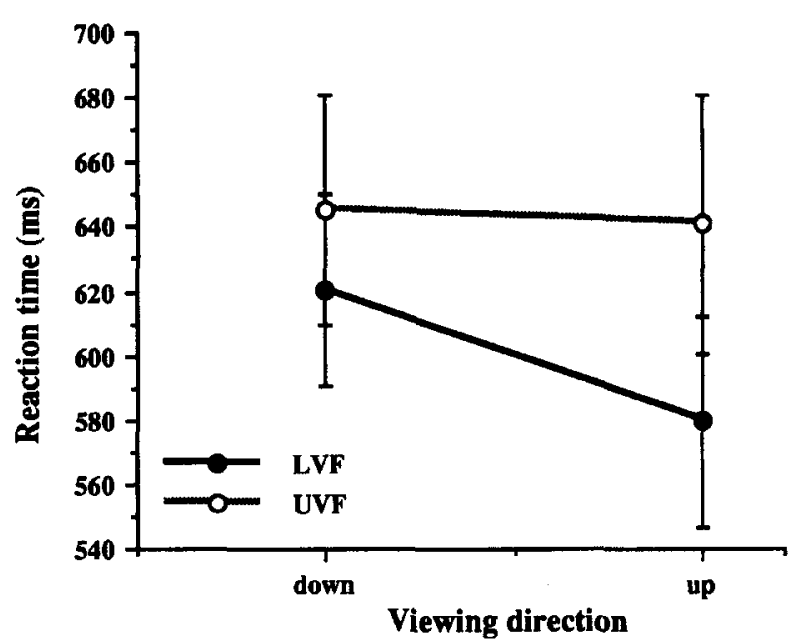

Figure 7. The asymmetry of viewing direction for lower visual field (LVF) and upper visual field (UVF) for the stimuli shown in Figure 6C. Means for 7 observers are graphed with their standard ermors.

reach significance. Similar results were obtained when mean RTs or the slopes of the RT-display-size relationship were used in the analyses instead. No statistical differences were obtained for viewing direction or visual field. These findings suggest that the increased size of the stimuli may have weakened the 3-D characteristics of the objects. This possibility is supported by the observers' reports that the large cubes were easily seen as separate features or feature groups in different configurations that were generally not 3-D. The absence of a visual field effect in this experiment is analogous to the findings of Experiment 1.

\section{EXPERIMENT 4}

From the previous experiments, it appears that the strength of the 3-D appearance of the stimulus objects may be important not only in determining the asymmetry of viewing direction, but also in determining the possible anisotropy favoring the LVF, both in terms of search asymmetry and in overall response latency. In Experiment 4 , the 3-D quality of the stimulus cubes was enhanced by adding perspective and shading.

\section{Method}

Subjects. The observers included the 2 authors and the same 5 undergraduate psychology students, all with normal or correctedto-normal vision (20/20 Snellen).

Stimuli and Procedure. The stimuli that were used in this experiment are shown in Figures 6B and 6C. The large cubes in Experiment 3 (see Figure $6 \mathrm{~A}$ ) had little perspective, and the implication that they were $3-D$ was not convincing. To make the $3-D$ nature of the stimulus objects more convincing, we introduced strong perspective so that the front surface was larger than the back surface and the sides were adjusted correspondingly. Their apparent length in depth was also increased, but the actual overall size remained the same as that in Experiment 3 ( $4^{\circ}$ vertically and horizontally). To further increase the effect, the cubes were not drawn in outline, but their sides were shaded solidly instead. In Part 1 (shown in Figure $6 \mathrm{~B}$ ), shading was not consistent with the same light source po- 
sition for both stimuli and thus did not reinforce the different vertical surface layout given by the different tilts. In Part 2 (Figure $6 \mathrm{C}$ ), on the other hand, the light source was always located at the top, and the shading was consistent with the tilt of the stimuli, thus reinforcing their particular orientations in 3-D space.

The procedure was the same as that used in the previous experiments. In particular, the display area was the same size as the area in Experiment 3 (a rectangle of $16^{\circ} \times 11^{\circ}$ of visual angle), with fixation points in the center just below and above. For the LVF conditions the upper cross was fixated, and for the UVF conditions the lower cross was fixated. There were two viewing directions with the target tilted either downward or upward. These four $(2 \times 2)$ conditions were presented to the observers in different randomized orders. Again, the displays consisted of 2, 6, or 10 items. The two parts were run as two separate experiments.

\section{Results and Discussion}

For both parts, a four-factor within-subjects ANOVA was performed on the RT data. In Part 1 , viewing direction $[F(1,6)=0.2, p>.67]$ and its interaction with visual field $[F(1,6)=0.09, p>.78]$ were not significant, but they showed trends in the same directions found in the previous experiments - upward-tilted targets gave faster responses, and the difference between the two viewing directions was bigger in the LVF. There was, however, a significant visual field effect $[F(1,6)=8.91, p<.025]$; responses to targets in the LVF were faster than those to targets in the UVF ( 603 vs. $657 \mathrm{msec}$ ).

In Part 2, overall viewing direction was not significant $[F(1,6)=3.25, p>.12]$, but its interaction with visual field $[F(1,6)=7.77, p<.032]$ and visual field itself $[F(1,6)=17.36, p<.006]$ were statistically significant. The interaction is graphed in Figure 7, which shows that the results for the two viewing directions were practically identical in the UVF, while the asymmetry was clearly present in the LVF. It is also clear that responses in the LVF were again faster than those in the UVF ( 600 vs. $643 \mathrm{msec}$ ). These results replicate the search asymmetry and the visual field anisotropies found in the previous experiments and demonstrate that the addition of more convincing 3-D qualities (perspective and shading) can bring out these effects, even for larger stimuli and when stimuli are confined to only one visual field.

\section{GENERAL DISCUSSION}

In the present experiments, we demonstrated an asymmetry of visual search that pertains to the orientation of stimulus objects in 3-D space in relation to the observer. Since the important geometric parameter is the relative orientation, this can be expressed either in terms of the orientation of the objects as seen by the observer (i.e., tilted downward or upward), or in terms of the observer's viewing direction (i.e., looking down onto or up at the objects). We investigated some conditions under which this asymmetry occurs and found two associated visual field anisotropies.

The results from these experiments can be interpreted to mean that the situation in which the observer looks at 3-D objects from above is a situation that is encountered more often, and therefore it is processed with more ease and against which a differently oriented object (i.e., one that is seen from below) stands out. On the other hand, the reverse arrangement, in which the observer views the objects from below, constitutes a less usual situation that needs more processing resources, and against which a target that is viewed from above is not so readily discriminated. This asymmetry was stronger in the LVF than in the UVF, and processing was often faster in the LVF.

\section{Methodological Considerations}

Two points need to be discussed with respect to the present methodology. The first concerns the possibility that the visual field anisotropies might be the result of uncontrolled eye movements. Even though fixation was required in all the experiments, eye movements were not recorded. The experimenter, however, visually checked the observers' compliance and reminded them not to use eye movements for the search. It is clear that this does not constitute an adequate means to control fixation. Small random deviations from the given fixation point, which most certainly occurred, are not crucial to the present arguments since the stimulated visual field regions would essentially remain the same. Larger random deviations from fixation would have had the effect of reducing the clear-cut separation of stimulus presentation in the different visual field conditions, and thus of reducing the search differences between the two visual fields both in terms of the overall RT and for the viewing direction asymmetry. The results of our experiments, however, show that the occurrence of visual field effects seems to depend more on other parameters, such as the perceived 3-D quality of the stimuli. On the other hand, a systematic downward slippage of fixation could explain the overall lower field advantage (as pointed out by one of the reviewers). This anisotropy occurred clearly only when the stimuli had a strong 3-D appearance, as in Experiment 4. From our observations and experience, moreover, such a systematic eye movement effect was not present in the present situation. In all the experiments except Experiment 1, the different visual field conditions were presented in separate trial runs, so that the observer knew in which field the target (and the distractors) would appear, which would have facilitated systematic eye movement effects. The asymmetry was found to differ in the two visual fields in Experiment 4, but not in Experiment 2. In Experiment 1, however, the observer could not predict the location of the target, yet in this experiment the effect of viewing direction differed between the two visual fields. It is therefore not likely that the anisotropy with respect to the asymmetry is the result of different eye movement patterns.

The second point concerns the strength of the 3-D appearance of the objects and its effect on the anisotropies. This factor was not varied systematically in the present experiments, nor was it measured independently. Yet this factor emerged as an important determinant for the two anisotropies. In Experiment 1, the outlined 3-D objects 
that resembled cubes were compared with similar objects that had no cues. Since it is impossible to make the 2-D and 3-D stimuli identical, except for the extent of 3-D appearance, it is not known if other geometric factors (e.g., different angles) contributed to the obtained differences in visual search for the two kinds of stimuli. Even though it is not known how salient the 3-D nature of the cubes was for the observers, it can be assumed that at least part of the time they were seen as cubes, especially since the observers referred to them as such. At any rate, only the 3-D stimuli showed an asymmetry effect of viewing direction, and this effect was stronger in the LVF. In Experiment 3, the observers found it more difficult to see the larger stimuli as 3-D cubes, and no anisotropies occurred. On the other hand, when the 3-D quality of the stimuli was increased by introducing linear perspective and shading in Experiment 4, the visual field anisotropies appeared - always favoring the LVFboth in faster overall RT and in a stronger asymmetry. In conclusion, perceived 3-D quality of the stimuli seems to be a prerequisite for the occurrence of the present visual field anisotropies.

\section{Asymmetry of Viewing Direction}

The main result of the present study is the demonstration of a search asymmetry for viewing direction. That the obtained asymmetry is not only the result of some arbitrary arrangement of the three surfaces that make up the stimulus, but also depends on the particular orientation of the object in 3-D space, is supported by the absence of an asymmetry for corresponding 2-D stimuli in Experiment 1. When linear perspective as a cue to dimension is taken away, the stimuli appear to be flat and show no asymmetry. An important limitation of the generality of the effect is suggested by the absence of an asymmetry in Experiment 3, where the size of the 3-D objects was increased. This suggests that the viewing direction asymmetry may be dependent on the strength of the induced 3-D percept, as discussed above. This conclusion is based only on informal comments by the observers, and it needs to be investigated systematically in further studies. It is supported, however, by the results of Experiment 4, where increased perspective and shading facilitated the asymmetry.

The appearance of the asymmetry also depended on which part of the visual field was stimulated. Such an anisotropy is interesting for the interpretation of the search asymmetry. The anisotropy was found when trials with targets presented in the LVF were intermingled with trials with targets in the UVF (Experiment 1), but disappeared when LVF and UVF trials were separated (Experiment 2). In the former case, the observer could not predict in which field the target would appear, but could do so in the latter case. Thus, the anisotropy occurred only when the task was more challenging. Then, an upward-tilted target was processed faster in the LVF than either a downward-tilted target in the LVF or both kinds of targets in the UVF, which were all about equal. Thus, this asymmetry signifies an advantage for the LVF - a fact that might be a clue to the reason for its existence.

\section{Theoretical Considerations}

The results of the present experiments may be explained by a built-in mechanism that might function along the same lines as the direction-of-shading mechanism, investigated by Kleffner and Ramachandran (1992) and Aks and Enns (1992); it is difficult to explain the resulting asymmetry by the traditional view (Treisman \& Gormican, 1988; see critique by Green, 1991). Treisman and Gormican's (1988) account for search asymmetries exploits the idea of the presence rather than the absence of an additional feature that distinguishes the target from the background stimuli. An example supporting such an account is a search for a "Q" (which corresponds to an "O" with an extra oblique bar) among Os, which is faster than a search for an O among Qs. Kleffner and Ramachandran referred to this account when explaining the asymmetry that they found for concave and convex objects. Briefly, they reported that concave objects defined by a shading gradient (dark at the top, light at the bottom) against a background of similarly defined convex objects (reverse gradient: light at the top, dark at the bottom) were easier to detect than objects that were presented when the situation was reversed. To fit the added-feature account, they hypothesized that the additional feature inherent in the concave objects could be depth reversal. This is because convex objects are more frequently encountered and thus constitute the norm, whereas concave objects are convex ones plus a reversal in depth.

This view, though satisfying in many cases, does not easily account for the present observations, nor is it very convincing in the case of the shaded objects. Such an interpretation applied to our results would suggest that the extra distinctive feature for upward-tilted objects would be an extra rotation in 3-D space to change the more frequently encountered downward-tilted objects into upward-tilted ones. Following this kind of explanation leads us to a possible neuropsychological account of the search asymmetry. The added rotation that must be performed is done well only in the LVF, which produces the asymmetry there. It has been shown that the parietal lobe, which is critical for mental rotations, also has a stronger relationship to the LVF (Previc, 1990). Therefore, the asymmetry is the result of the specialized function of the LVF.

An alternate explanation would be one based on ecological considerations. Most objects that would be encountered during locomotion, and that either have to be avoided or attended to, would be smaller than ourselves and located below eye level. We would therefore most likely look down onto them (or they would appear tilted downward from our point of view). They would, there- 
fore, also appear predominantly in the LVF. It would be of survival value to detect a differently oriented object quickly and preattentively. Thus, the asymmetry is the result of an adaptation of the early visual system to ecological pressure.

A third explanation would be an experiential one, arguing basically along the same lines as the ecological explanation, but proposing instead that the individual acquires the asymmetry during development as a result of the specific interaction with the environment. The present experiments were not designed to discriminate between these possibilities, though the ecological explanation is our preferred approach.

At a more detailed level, one might wonder about the mechanisms that exist to build up perceptual objects from features or cues. Aks and Enns (1992) report the finding that outline shape, type of shading, and background luminance contribute relatively equally as cues to object depth. As argued, the search processes are sensitive to precursor descriptions of the 3-D representation and not to the representation itself. The visual system at that stage, therefore, is said to be looking for predictors (Enns \& Rensink, 1991), which will allow further integration to occur if these predictors are not in conflict. This view emphasizes a statistical component in the visual processing that could be inherited or acquired through development and/or experience. Our present observations suggest that the addition of more depth cues, such as shading and increased perspective, makes the asymmetry more prominent, as long as they serve to increase the perceived 3-D quality of the objects. Further observations are necessary, however, to determine the types of interaction that exist between the different cues. In conclusion, our studies show that early visual processing makes use of heuristics that take into account specific relationships between the relative locations of observer and 3-D objects.

\section{REFERENCES}

AKs, D. J., \& ENNs, J. T. (1992). Visual search for direction of shading is influenced by apparent depth. Perception \& Psychophysics, 52, 63-74.

ENNS, J. T., Ochs, E. P., \& RENSINK, R. A. (1990). VSearch: Macintosh software for experiments in visual search. Behavior Research Methods, Instruments, \& Computers, 22, 118-122.

ENNS, J. T., \& RENSINK, R. A. (1990). Influence of scene-based properties on visual search. Science, 247, 721-723.

EnNs, J. T., \& Rensink, R. A. (1991). Preattentive recovery of three dimensional orientation from line drawings. Psychological Review, 98, 335-351.

GreEN, M. (1991). Visual search, visual streams, and visual architectures. Perception \& Psychophysics, 50, 388-403.

KlefFNeR, D. A., \& Ramachandran, V. S. (1992). On the perception of shape from shading. Perception \& Psychophysics, 52, 18-36.

Previc, F. H. (1990). Functional specialization in the lower and upper visual fields in humans: Its ecological origins and neurophysiological implications. Behavioral \& Brain Sciences, 13, 519-575.

Ramachandran, V. (1988). Perceiving shape from shading. Scientific American, 259, 76-83.

RAMACHANDRAN, V. (1989). Interactions between motion, depth, color and form: The utilitarian theory of perception. In C. Blakemore (Ed.), Vision: Coding and efficiency (pp. 346-360). Cambridge: Cambridge University Press.

TownSEND, J. (1972). Some results concerning the identifiability of parallel and serial processes. British Journal of Mathematical \& Statistical Psychology, 25, 168-199.

Treisman, A., \& GoRmican, S. (1988). Feature analysis in early vision: Evidence from search asymmetries. Psychological Review, 95, 15-48.

Treisman, A., \& Souther, J. (1985). Search asymmetry: A diagnostic for preattentive processing of separable features. Journal of Experimental Psychology: General, 114, 285-310.

Wolfe, J. M., CaVe, K. R., \& Franzel, S. L. (1989). Guided search: An alternative to the modified feature integration model for visual search. Journal of Experimental Psychology: Human Perception \& Performance, 15, 419-433.

(Manuscript received October 20, 1992; revision accepted for publication January 18,1994 .) 